\title{
Disseminated Cryptococcosis- Induced Skin Ulcers in a Patient with Autoimmune Hepatitis
}

\author{
Takaharu Ikeda Chikako Kaminaka Yuki Yamamoto Fukumi Furukawa \\ Department of Dermatology, Wakayama Medical University, Wakayama, Japan
}

\section{Key Words}

Autoimmune hepatitis · Cryptococcosis · Skin ulcer

\begin{abstract}
We report the case of a 68-year-old woman with autoimmune hepatitis (AIH) who had leg ulcers induced by disseminated cryptococcosis. She had received prednisolone for her AIH at $20 \mathrm{mg} /$ day for maintenance. On the initial visit, she complained of a painful ulcer that had round, shallow pockets with erythema and erythematous subcutaneous indurations on the right thigh. Several metacarpophalangeal joints and wrist joints were swollen, with tenderness and stiffness in the morning for over $3 \mathrm{~h}$. Her serum rheumatoid factor was high. Since other autoimmune disorders such as rheumatoid arthritis can present with $\mathrm{AIH}_{\text {, it was }}$ necessary to distinguish it from ulcers due to rheumatoid arthritis, although the characteristic features of these ulcers seemed to be different. A biopsy specimen from the erythematous skin showed globe-shaped organisms in the dermis and subcutaneous tissues; vasculitis and phlebostasis were not observed. The results from computed tomography scans and sputum culture led to the diagnosis of disseminated cryptococcosis. The administration of fluconazole, fosfluconazole, and voriconazole for about 2 months improved the cryptococcal pneumonia, but the size of the skin ulcer enlarged. The administration was changed to itraconazole, which reduced the size. Cryptococcal infections occur more commonly in immunocompromised hosts, including patients under immunosuppressive therapies such as corticosteroids. The possibility that the skin ulcers in immunocompromised hosts may be caused by cryptococcosis should be considered.


Ikeda et al.: Disseminated Cryptococcosis-Induced Skin Ulcers in a Patient with Autoimmune Hepatitis

\section{Introduction}

Cryptococcus neoformans is a yeast-like encapsulated fungus, which is mainly found in the droppings of pigeons. The inhalation of contaminated dust is the major infectious pathway, which can result in pulmonary involvement and may spread to other organs such as the CNS and skin. Cryptococcal infections occur more commonly in immunocompromised hosts who have predisposing factors, such as acquired immunodeficiency syndrome or immunosuppressive therapies including corticosteroids for systemic lupus erythematosus, rheumatoid arthritis (RA), etc. [1-3].

Autoimmune hepatitis (AIH), whose most common symptom is fatigue but is often asymptomatic, is characterized by hepatosplenomegaly, hypergammaglobulinemia, antismooth muscle antibodies, or anti-liver/kidney microsome 1 antibodies [4]. Antinuclear antibodies, rheumatoid factor (RF), and anti-cyclic citrullinated peptide (CCP) antibodies may be detected [5]. The common extrahepatic manifestations are arthralgia, non-erosive arthritis mostly on the small joints, and myalgia [4, 6]. Furthermore, AIH cases can have other concurrent autoimmune disorders such as RA, autoimmune thyroiditis, type 1 diabetes, or ulcerative colitis. The standard treatments are oral corticosteroids alone or in combination with immunosuppressant such as azathioprine.

Here, we report on a case with AIH and leg ulcers caused by disseminated cryptococcosis. Since the patient had polyarthritis which mimicked RA symptomatically, it was necessary to distinguish it from ulcers due to RA.

\section{Case Report}

A 68-year-old woman consulted our department for skin ulcers on her right thigh complicated with suspected RA. She had been diagnosed with type 1 diabetes and Hashimoto's disease 15 years ago, and with AIH and Sjögren's syndrome 3 years ago, and was eventually diagnosed with autoimmune polyendocrinopathy syndrome. She received prednisolone at $30 \mathrm{mg} /$ day as the first treatment for AIH. The dose of the corticosteroids was difficult to taper due to arthralgia, and she continued to take medication at $20 \mathrm{mg} /$ day as a maintenance dose. At her initial visit, the metacarpophalangeal joints of her bilateral forefingers and middle fingers, and her wrist joints were swollen with tenderness. She also complained that the morning stiffness of the fingers persisted for more than $3 \mathrm{~h}$. One month prior to the initial visit, a skin ulcer appeared on the lateral side of the right thigh. She was hospitalized for the ulcer due to suspected RA, and high levels of serum RF and matrix metalloproteinaseIII were administered.

On hospitalization, her vital signs were as follows: temperature $37.1^{\circ} \mathrm{C}$, blood pressure $120 / 64 \mathrm{~mm} \mathrm{Hg}$, pulse rate 64 beats/min, and oxygen saturation $97 \%$. She had a productive cough and her legs were edematous. The physical examination revealed some fine crackles by stethoscope. There was a painful ulcer that had round, shallow pockets with erythema and erythematous subcutaneous indurations on the right thigh (fig. 1).

The main laboratory findings were as follows: white blood cell count 13,500/ $\mu \mathrm{l}$ (normal range 3,500-9,800), creatinine $1.42 \mathrm{mg} / \mathrm{dl}$ (normal range 0.47-0.72), urea nitrogen 35 $\mathrm{mg} / \mathrm{dl}$ (normal range 10-20), C-reactive protein (CRP) $4.29 \mathrm{mg} / \mathrm{ml}$ (normal range <0.03), brain natriuretic peptides $752.5 \mathrm{pg} / \mathrm{ml}$ (normal range $<18.4$ ), $\mathrm{RF} 117.0 \mathrm{U} / \mathrm{ml}$ (normal range $<19$ ), anti-CCP antibody $16.5 \mathrm{U} / \mathrm{ml}$ (normal range <4.5), CH50 $58 \mathrm{U} / \mathrm{ml}$ (normal range 3050), KL-6 $257 \mathrm{U} / \mathrm{ml}$ (normal range $<500$ ) [1-3], and $\beta$-D glucan $6 \mathrm{pg} / \mathrm{ml}$ (normal range 011). 
Ikeda et al.: Disseminated Cryptococcosis-Induced Skin Ulcers in a Patient with Autoimmune Hepatitis

Before hospitalization, a chest X-ray and computed tomography (CT) scan revealed cardiomegaly and some old linear shadows, but no shadows suggestive of pneumonia and pleural effusion were observed. An echocardiogram showed a decline in the left heart function but no signs of heart failure. We prescribed diuretics for the leg edema.

Although the characteristic features of the ulcers seemed to be different, it was possible that the cause of the skin ulcers was RA, which complicated the AIH, because the RF value was high, the anti-CCP antibodies were positive, and her articular symptoms mimicked RA.

A blood culture was negative, but a sputum culture was positive for Pseudomonas aeruginosa and a urine culture was positive for Proteus mirabilis. A biopsy specimen showed granulomatous inflammation, degenerative necrosis, and globe-shaped organisms in the dermis and subcutaneous tissues (fig. 2). We began to treat her with ceftazidime intravenously and fluconazole orally at a starting dose of $200 \mathrm{mg} /$ day and decreased to $100 \mathrm{mg} /$ day which was adjusted to the patient's decreased renal function. The serum latex agglutination titer to Cryptococcus, which was measured after the result from the skin biopsy, was 1:4,096. The follow-up CT scan revealed a new nodule on the lower right lung, and a small amount of pleural effusion, and the follow-up sputum culture was positive for Cryptococcus neoformans. However, the sensitivity of the antifungal drugs could not be examined because the amount of Cryptococcus detected was so small. Therefore, we diagnosed it as disseminated cryptococcosis. We asked her about having had contact with pigeons, but she denied. We got the result of the follow-up sputum culture after the start of the treatment with antifungal drugs. The detected amount of Cryptococcus in this culture was very small. Consequently, we considered that we could not get meaningful results for the further cultures under that administration. Bronchoscopy and a lumbar puncture could not be performed because her general conditions worsened. The oral fluconazole was changed to fosfluconazole intravenously at an initial dose of $200 \mathrm{mg} /$ day and a maintenance dose of $100 \mathrm{mg} /$ day. Her CRP levels decreased to nearly within normal limits, and the antifungal agent was changed to oral $100 \mathrm{mg} /$ day fluconazole again. However, thrombocytopenia aggravated and her renal function worsened, and the agent was changed again to oral voriconazole at a dose of 600 $\mathrm{mg} /$ day and thereafter $300 \mathrm{mg} /$ day as maintenance dose. Although repeated CT scans showed that the nodule disappeared and the CRP levels did not increase due to the skin involvements, the indurations progressed to ulcerations and their size enlarged. After she was transferred to another hospital, the drug was changed to itraconazole, which clearly reduced the skin manifestations.

\section{Discussion}

AIH is a chronic necroinflammatory liver disorder of unknown etiology. The common symptom is fatigue, but it is often asymptomatic, and the articular manifestations may occur as arthralgia and non-erosive arthritis on the small joints [4, 6]. Although ANA, RF, anti-CCP antibodies and so on can be detected in cases of AIH, and it can present with other autoimmune disorders such as autoimmune thyroiditis, type 1 diabetes, ulcerative colitis, and RA $[4,5]$.

Anti-CCP antibodies were detected in $9-11 \%$ of the type 1 AIH cases [7-9]. Fusconi et al. [7] reported no significant differences in the prevalence of rheumatic manifestations between patients positive versus negative for these antibodies. However, Montano-Loza et al. [8] and Koga et al. [9] showed that seropositivity for anti-CCP antibodies was associated with a higher frequency of RA, and that anti-CCP antibodies might be helpful markers for diagnosing concomitant RA in cases of AIH. In our case, the anti-CCP antibodies were 
Ikeda et al.: Disseminated Cryptococcosis-Induced Skin Ulcers in a Patient with Autoimmune Hepatitis

positive but had low titers, and the high RF value plus her articular symptoms led to the suspicion of RA. Therefore, we considered the possibility that the leg ulcers were caused by concurrent RA, although the characteristic features of her leg ulcers seemed to be different from those typical of RA.

Leg ulceration in RA is not uncommon, and the overall prevalence is reported to be about $10 \%[10,11]$. One of the main causes for forming ulceration due to RA is rheumatoid arteritis. Arteritic ulcers are usually deep and punched out lesions, and are characterized by the absence of venous manifestations and the presence of seropositivity for RF, often at high titers [12]. On the other hand, skin involvements were reported to occur in $10-20 \%$ of disseminated cryptococcosis cases negative for human immunodeficiency virus [1]. Cutaneous manifestations can take various forms such as ulcers, papules, pustules, abscesses, purpura, nodules, subcutaneous swellings, and so on. To make a definitive diagnosis of cryptococcosis, the results of skin biopsies from the eruptions are important.

In our case, the skin biopsy specimens showed no vasculitis, and there were globe-like organisms in the dermis and subcutaneous tissues. Taken together, the additional CT findings, the high titer of latex agglutination to Cryptococcus, and the follow-up sputum culture all resulted in the diagnosis of disseminated cryptococcosis. In general, the possibility that skin ulcers in immunocompromised hosts may be caused by cryptococcosis should be considered.

\section{Disclosure Statement}

The authors have no conflicts of interest to disclose.

\section{References}

1 Pema K, Disz J, Guerra LG, Verghese A: Disseminated cutaneous cryptococcus. Comparison of clinical manifestations in the pre-AIDS and AIDS eras. Arch Intern Med 1994;154:1032-1034.

-2 Chen HS, Tsai WP, Leu HS, Ho HH, Liou LB: Invasive fungal infection in systemic lupus erythematosus: an analysis of 15 cases and a literature review. Rheumatology 2007;46:539-544.

-3 Lu HC, Yang YY, Huang YL, Chen TL, Chuang CL, Lee FY, Lee SD: Disseminated cryptococcosis initially presenting as cellulitis in a rheumatoid arthritis patients. J Chin Med Assoc 2007;70:249-252.

Edward LK: Autoimmune hepatitis. N Engl J Med 2006;354:54-66.

5 Czaja AJ: Autoimmune liver disease and rheumatic manifestations. Curr Opin Rheumatol 2007;19:74-80. Loukili NH, Pettorin C, Noel E, Andres E: Type 1 autoimmune hepatitis revealed by a dysphonia related to cricoarytenoid arthritis. QJM 2003;96:171-172.

-7 Fusconi M, Vannini A, Dall'aglio AC, Pappas G, Cassani F, Ballardini G, Frisoni M, Grassi A, Bianchi FB, Zauli D: Anti-cyclic citrullinated peptide antibodies in type 1 autoimmune hepatitis. Aliment Pharmacol Ther 2005;22:951-955.

8 Montano-Loza A, Craja AJ, Carpenter HA, Piette A, Murphy D, Shums Z, Burlingame R, Norman GL: Frequency and significance of antibodies to cyclic citrullinated peptide in type 1 autoimmune hepatitis. Autoimmunity 2006;39:341-348.

-9 Koga T, Migita K, Miyashita T, Maeda Y, Nakamura M, Abiru S, Myoji M, Komori A, Yatsuhashi H, Eguchi K, Ishibashi H: Determination of anti-cyclic citrullinated peptide antibodies in the sera of patients with liver disease. Clin Exp Rheumatol 2008;26:121-124.

10 Matricali GA, Boonen A, Verduyckt J, Taelman V, Verschueren P, Sileghem A, Corluy L, Westhovens R.: The presence of forefoot problems and the role of surgery in patients with rheumatoid arthritis. Ann Rheum Dis 2006;65:1254-1255.

-11 Firth J, Hale C, Helliwell P, Hill J, Nelson EA: The prevalence of foot ulceration in patients with rheumatoid arthritis. Arthritis Rheum 2008;59:200-205.

$\$ 12$ Mortimer PS, Burnand KG: Diseases of the veins and arteries: leg ulcers; in Burns T, Breathnach S, Cox N, Griffiths C (eds): Rook's Textbook of Dermatology, ed 7. Massachusetts, Blackwell Science Ltd., 2004, p 50.36 . 


\section{Case Reports in Dermatology}

\section{Case Rep Dermatol 2014;6:98-102}

DOI: $10.1159 / 000360978$

Ikeda et al.: Disseminated Cryptococcosis-Induced Skin Ulcers in a Patient with
Autoimmune Hepatitis

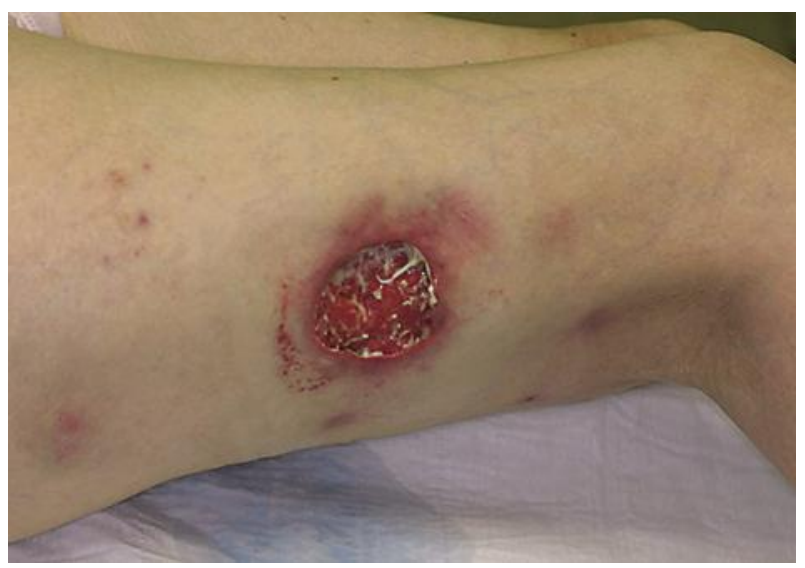

Fig. 1. Clinical findings at the first visit. A painful ulcer with round, shallow pockets and some indurations with redness on the lateral side of the right thigh is shown.

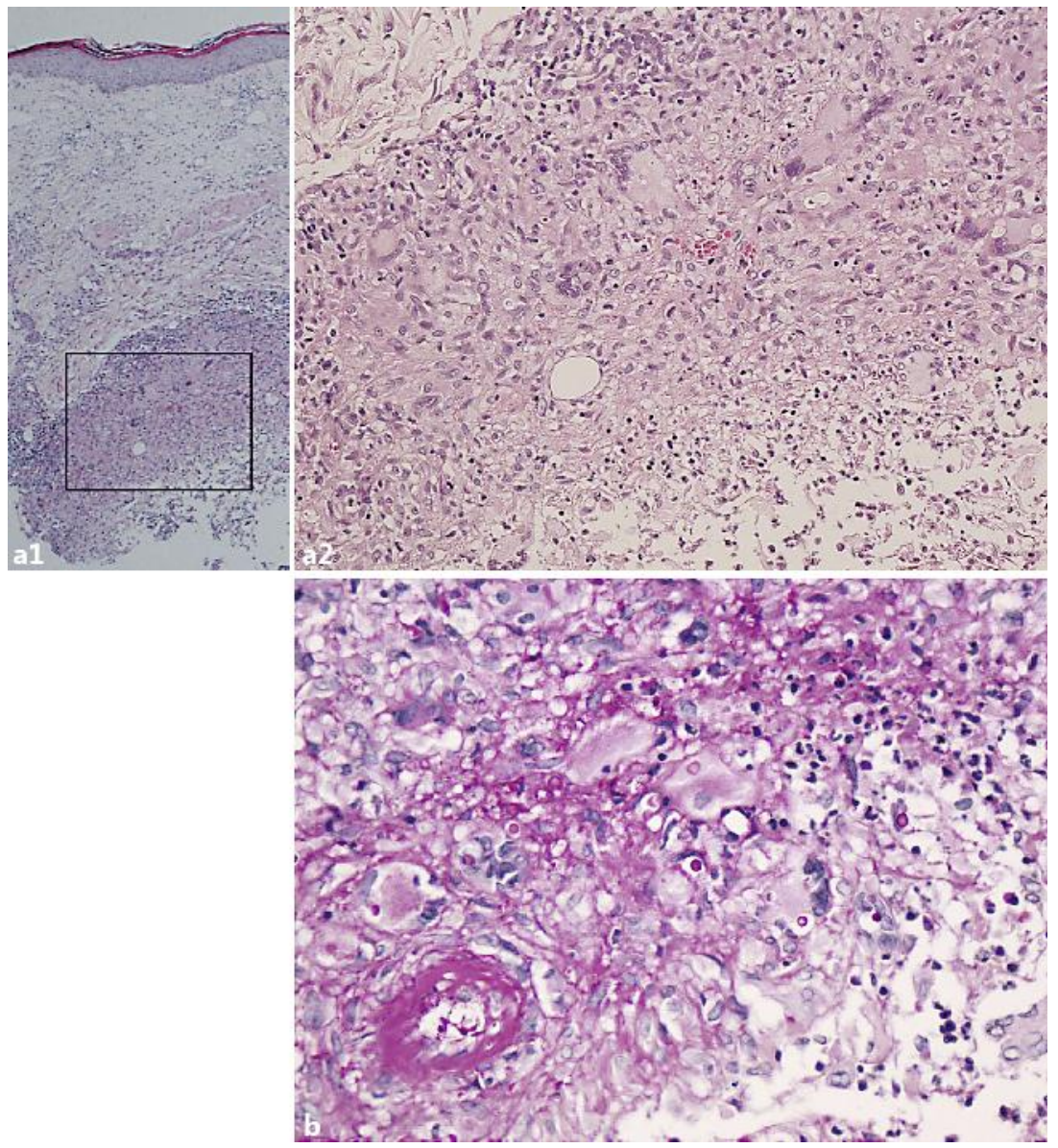

Fig. 2. Histopathological findings of the biopsy specimen from the outline of the skin ulcer. Granuloma with multinucleated giant cells, degenerative necrosis, and globe-like organisms in the lower dermis and subcutaneous tissues is shown. a Hematoxylin-eosin stain, $\times 40$ and $\times 200$. b PAS stain of the subcutaneous tissue, $\times 400$. 